\title{
CDISC SDTM Graft Type Terminology
}

National Cancer Institute

\section{Source}

National Cancer Institute. CDISC SDTM Graft Type Terminology. NCI Thesaurus. Code C117737.

Terminology associated with the graft type codelist of the Clinical Data Interchange Standards Consortium (CDISC) Study Data Tabulation Model (SDT M). 